Published in final edited form as:

J Arthroplasty. 2013 October ; 28(9): . doi:10.1016/j.arth.2013.06.011.

\title{
Topical tranexamic acid reduces blood loss and transfusion rates in total hip and total knee arthroplasty
}

\author{
Gerhardt Konig ${ }^{*}$ Brian R. Hamlin^, and Jonathan H. Waters\# \\ ${ }^{\star}$ Department of Anesthesiology, University of Pittsburgh School of Medicine \\ ${ }^{\wedge}$ The Bone and Joint Center, Magee-Womens Hospital of the University of Pittsburgh Medical \\ Center \\ \#Department of Anesthesiology, Magee-Womens Hospital of the University of Pittsburgh Medical \\ Center \# The McGowan Institute For Regenerative Medicine, University of Pittsburgh, Pittsburgh, \\ $\mathrm{Pa}$
}

\begin{abstract}
The objective of this study was to determine if tranexamic acid (TXA) applied topically reduced postoperative bleeding and transfusion rates after primary total hip arthroplasty (THA) and primary total knee arthroplasty (TKA).

Two hundred and ninety consecutive patients from a single surgeon were enrolled. In TKA, TXA solution was injected into the knee after closure of the arthrotomy. In THA, the joint was bathed in TXA solution at three points during the procedure. In both THA and TKA the TXA solution was at a concentration of $3 \mathrm{gm}$ TXA per $100 \mathrm{~mL}$ saline.

The mean blood loss was significantly higher in the non-TXA patients in both TKA and THA groups. Postoperative transfusions decreased dramatically with TXA, dropping from $10 \%$ to $0 \%$, and from $15 \%$ to $1 \%$, in the TKA and THA groups, respectively.

Topical application of TXA significantly reduces postoperative blood loss and transfusion risk in TKA and THA.
\end{abstract}

\section{Keywords}

Blood Transfusion; Tranexamic Acid; Antifibrinolytic; Total Knee Arthroplasty; Total Hip Arthroplasty

\footnotetext{
(C) 2013 Elsevier Inc. All rights reserved

Gerhardt Konig, MD (Corresponding author), University of Pittsburgh School of Medicine Department of Anesthesiology, MageeWomens Hospital of UPMC, 300 Halket St., Suite 3510, Pittsburgh, PA 15213, Phone: (412) 641-4260, Fax: (412) 641-4766, konigg@ upmc.edu; Brian R. Hamlin, MD, Magee-Womens Hospital of UPMC The Bone and Joint Center 300 Halket Street, Suite 1601 Pittsburgh, PA 15213; Jonathan H. Waters, MD Magee-Womens Hospital of UPMC Department of Anesthesiology 300 Halket St., Suite 3510 Pittsburgh, PA 15213.

Conflicts of Interest: none pertaining to the material in this manuscript

Publisher's Disclaimer: This is a PDF file of an unedited manuscript that has been accepted for publication. As a service to our customers we are providing this early version of the manuscript. The manuscript will undergo copyediting, typesetting, and review of the resulting proof before it is published in its final citable form. Please note that during the production process errors may be discovered which could affect the content, and all legal disclaimers that apply to the journal pertain.
} 


\section{Introduction}

Total joint replacement surgery is associated with large amounts of perioperative blood loss and significant rates of transfusions. Patients undergoing total knee arthroplasty (TKA) are transfused at rates of $11-21 \%$, and patients undergoing total hip arthroplasty (THA) are transfused at rates of $16-37 \%{ }^{1}$. Antifibrinolytics have been used in orthopedic surgery via an intravenous (IV) route, resulting in a 50\% reduction in the rate of transfusions ${ }^{2-4}$. However, there have been isolated case reports of thrombus formation, which has generated concerns over the risk of thromboembolic complications in a patient population already at high risk for deep vein thrombosis and pulmonary embolism. This has prevented the widespread acceptance of the use of IV antifibrinolytics in total joint replacement surgery 5,6 . Topical application of antifibrinolytics may produce the same efficacy, but with much lower systemic absorption and thus much lower risk for thromboembolic complications. Prior studies have suggested that topical tranexamic acid (TXA), an antifibrinolytic, is safe and effective at reducing postoperative bleeding in orthopedic procedures ${ }^{7,8}$. These studies were small and limited to screw fixation of the lumbar spine and total knee arthroplasty (TKA). The objective of this prospective comparative study was to determine if topical tranexamic acid (TXA) reduced postoperative bleeding and transfusion rates after primary total hip arthroplasty (THA) and primary TKA.

\section{Methods}

Following approval of the University of Pittsburgh Medical Center's institutional review board, all patients undergoing primary THA and TKA over an eighteen month period by a single surgeon at a single institution were enrolled. This study evaluated a process change within our blood management program. The TXA group consists of all patients for one year after introducing a topical TXA protocol, and the control group consists of all the patients in the six months prior to implementing a topical TXA protocol. The rest of the blood management protocol was consistent throughout all 18 months of the study period. All patients underwent preoperative anemia screening and were referred to their primary care physician for hemoglobin (Hgb) optimization if the Hgb level was less than 10. All patients undergoing THA or TKA in the TXA group were given topical TXA. Only patients undergoing primary THA or primary TKA were included in the analysis. The only exclusion criteria were allergy to TXA and revision surgery. Patient demographic data (age, sex) as well as general health (BMI, ASA physical status score) were collected. Preoperative Hgb data and daily postoperative $\mathrm{Hgb}$ data were collected. All transfusions were noted. Patients were managed with a standard blood management program, which included a strict transfusion Hgb trigger of 8.0 and the use of $500 \mathrm{~mL}$ hetastarch volume expansion for postoperative hypotension, dizziness, or lightheadedness prior to consideration of a transfusion. No changes to the surgical or postoperative treatment protocols were implemented during the study period that may have affected blood loss. All procedures were performed under spinal anesthesia.

All total hip arthroplasties were performed with a posterior approach. Major bleeders were identified and cauterized in a standard fashion. After acetabular preparation the acetabulum was bathed with $20 \mathrm{~mL}$ of the TXA solution at a concentration of $3 \mathrm{gm}$ TXA per $100 \mathrm{~mL}$ saline, as was found effective in a prior study. ${ }^{8}$ An uncemented acetabular component was then impacted. Adjunctive screw fixation was used in all cases with placement of one to two screws in the safe posterior superior quadrant. After femoral canal broach preparation, $20 \mathrm{~mL}$ of the TXA solution was placed within the femoral canal. The uncemented femoral stem was then impacted into place. After reduction of the final hip components, the remaining $60 \mathrm{~mL}$ of TXA fluid was poured into the wound. The TXA solution was left within the wound. The external rotators and capsule were repaired through drill holes in the trochanter in all cases. 
Deep fascia, subcutaneous, and skin closure were performed in a standard fashion in all cases. A drain was not used.

All total knee arthroplasties were performed through a standard medial parapatellar approach under tourniquet control. A posterior stabilized design was used and the patella resurfaced in all cases. After cementation of all components and placement of final polyethylene, the tourniquet was released. Hemostasis was then achieved followed by placement of a deep drain and closure of the arthrotomy. After clamping of the drain the knee was injected with $100 \mathrm{~mL}$ of the TXA solution $(3 \mathrm{gm}$ TXA/100mL saline). The remainder of the wound was then closed in standard fashion. The drain was clamped for one hour and then released. All drains were removed on the day following the surgery.

The primary outcomes were hospital length of stay (LOS), the nadir postoperative Hgb, maximum Hgb drop from preoperative levels, transfusion rates, and blood loss. Blood loss was calculated using equations described by Good et al. ${ }^{9}$ and Nadler et a ${ }^{10}$. Total blood loss is calculated from total hemoglobin loss $\left(\mathrm{Hgb}_{\mathrm{loss}}\right)$, which in turn is calculated from the difference between preoperative $\mathrm{Hgb}$ and the minimum hemoglobin during the hospital stay:

$$
\begin{aligned}
& \text { Blood loss (in } \mathrm{mL})=100 \mathrm{~mL} / \mathrm{dL} \times \mathrm{Hgb}_{\text {loss }} / \mathrm{Hgb}_{\mathrm{i}} \\
& \mathrm{Hgb}_{\text {loss }}=\mathrm{BV} \times\left(\mathrm{Hgb}_{\mathrm{i}}-\mathrm{Hgb}_{\mathrm{e}}\right) \times 10 \mathrm{dL} / \mathrm{L}+\mathrm{Hgb}_{\mathrm{t}} \\
& \mathrm{BV}=\text { Estimated total body blood volume in liters } \\
& =0.3669 \times \mathrm{H}^{3}+0.03219 \times \mathrm{W}+0.6041 \text { (for men), and } \\
& =0.3561 \times \mathrm{H}^{3}+0.03308 \times \mathrm{W}+0.1833 \text { (for women) } \\
& \mathrm{H}=\text { Height in meters } \\
& \mathrm{W}=\text { Body mass in } \mathrm{kg} \\
& \mathrm{Hgb} \\
& \mathrm{Hgb}_{\mathrm{e}}=\mathrm{Hgb} \text { concentration prior to surgery }(\mathrm{g} / \mathrm{dL} \text { ) } \\
& \mathrm{Hgb}_{\mathrm{t}}=\text { Total amount of allogeneic Hgb transfused }(\mathrm{g}) . \text { No patients had a nadir } \\
& \mathrm{hemoglobin}_{\mathrm{a}} \text { after they were transfused so this variable was dropped from the equation }
\end{aligned}
$$

All data was reported using summary statistics including means and standard deviations for quantitative data, and frequencies and percentages for qualitative data. The groups of continuous variables were compared using the unpaired two-tailed Student's T-test. P values of $<0.05$ were considered significant. A Fisher's Exact test was used for comparison of categorical variables.

\section{Results}

From September 2010 until March 2011 all patients undergoing total joint replacement did not receive any TXA. During this time 40 patients underwent primary unilateral THA, and 29 patients underwent primary unilateral TKA. All of these patients were included in the study. From March 2011 onwards, TXA was used in all cases. Between March 2011 and March 2012, 93 patients underwent primary unilateral THA, and 130 patients underwent primary unilateral TKA. All but two of these patients were included in the study. The two excluded patients had complications during surgery (an intraoperative fracture, and a subtrochanteric osteotomy).

There were no differences in age, body mass index (BMI), American Society of Anesthesiologists (ASA) status, or preoperative hemoglobin levels between the groups of patients that did and did not receive TXA (Table 1). 
Primary outcomes are listed in Table 2 . The mean blood loss was significantly more in the patients that did not receive TXA in both the TKA and THA surgeries. In the THA patients, the blood loss was $345 \mathrm{~mL}$ less when TXA was used, and in the TKA patients the blood loss was $231 \mathrm{~mL}$ less when TXA was used. The maximum in-hospital Hgb drop was also significantly lower in the TXA patients in both groups. The percentage of patients that required a transfusion postoperatively decreased dramatically in both TKA and THA groups, dropping from $10 \%$ to $0 \%$ in the TKA group when TXA was used ( 3 transfused out of 29 patients, as compared with 0 transfused out of 130 , respectively), and dropping from $15 \%$ to $1 \%$ in the THA group when TXA was used (6 transfused out of 40 patients, as compared with 1 transfused out of 91, respectively). We used a firm postoperative transfusion threshold of $\mathrm{Hgb}<8.0 \mathrm{~g} / \mathrm{dL}$, and all patients that received a transfusion fell below this level (range $6.8-7.9 \mathrm{~g} / \mathrm{dL}$ ). Hospital length of stay was also significantly shorter in patients in both surgical groups when TXA was used, dropping by 0.6 days in THA patients, and dropping by 0.4 days in TKA patients. No patients required an intraoperative blood transfusion. No patients had thromboembolic complications.

\section{Discussion}

Topical administration of TXA at the end of TKA and THA significantly reduced postoperative bleeding and risk for transfusion. The blood loss was 20 and $25 \%$ higher, respectively, in TKA and THA when TXA was not used. The drop in Hgb was 20 and 27\% higher, respectively, in TKA and THA when TXA was not used. This translated into a much lower risk of requiring a blood transfusion in both the TKA and THA patients in which TXA was used. None of the TKA patients with TXA required a transfusion whereas $10 \%$ of them did when TXA was not used. Only $1 \%$ of THA patients with TXA required a transfusion as compared with $15 \%$ that required one when TXA was not used. The same transfusion criteria were used during both phases of the study which was a Hgb trigger of $8.0 \mathrm{gm} / \mathrm{dL}$. No patients had thromboembolic complications.

Antifibrinolytics are a class of drugs that have been in use since the 1960s. ${ }^{2}$ TXA is an analog of the amino acid lysine. It competitively inhibits plasminogen activation and plasmin binding to fibrin, thus inhibiting fibrin degradation. ${ }^{5}$ Since it works by reducing breakdown of fibrin once formed, it is not procoagulant per se, but rather supportive of coagulation already in progress. This makes it potentially well suited for use in reducing postoperative bleeding, where surgical hemostasis has been achieved and fibrinolytic activity needs to be suppressed to help maintain hemostasis without promoting venous thrombus formation.

Tranexamic acid has been shown to reduce the risk of receiving transfusions by a third in a meta-analysis of all surgeries ${ }^{11}$. It has been used successfully in orthopedic surgery via an intravenous route, with several studies showing significant reductions in bleeding and risk of transfusion after TKA and THA ${ }^{12-20}$. However, concerns remain over the risk of thromboembolic complications after systemic administration ${ }^{5,6}$. Topical application leads to $70 \%$ lower systemic absorption, and may therefore be a safer alternative to giving it systemically ${ }^{8}$. Akizuki et al. ${ }^{21}$ first reported topical use of TXA in orthopedic surgery in 1997, reporting no postoperative blood transfusions in 42 simultaneous bilateral cementless TKA patients and 64 unilateral cementless TKA patients. The technique did not appeal to many surgeons, or they were not aware of it, because it was not until 2010 when another group reported on the use of topical TXA in TKA. Wong et al. ${ }^{8}$ performed a study with 124 patients and reported significantly reduced postoperative bleeding after TKA when TXA was applied topically to the wound before closure. Two more small studies were published in 2011, also showing reduced postoperative bleeding, but again not commenting on changes in rates of transfusions. ${ }^{22,} 23$ Another study in 2012 was performed by Mutsuzaki et 
al. ${ }^{24}$ in which they showed that total blood loss, total drainage, mean transfusion volume, and transfustion rates were all lower when TXA was injected through the drain after TKA and the drain then clamped, as compared with not injecting TXA. A problem with the interpretation of their data, however, is that they routinely used autotransfusion from preoperative autologous donation. The frequency of autotransfusion varied considerably among the groups, which could have influenced the results. The same year Seo et al. ${ }^{25}$ reported a comparison between intravenous TXA, topical TXA, and placebo in TKA. They showed significantly decreased blood loss and transfusion requirements in both TXA groups, with larger decreases seen with intraarticular TXA as compared with intravenous. Two limitations to this study were that it was done using extramedullary minimally invasive surgical techniques, which are difficult to compare to conventional TKA, and that their transfusion rates for all groups were much higher than what is usually reported in the literature (34\% for IV TXA, 20\% for topical TXA, and 94\% for placebo). It is harder to compare their results with other studies since their baseline transfusion rates are so high.

Our study is the first to report the changes in transfusion rates with a single surgeon for both TKA and THA before and after a topical TXA protocol was introduced. Blood transfusion rates dropped significantly in both TKA and THA cases, with no patients requiring transfusion after TKA and only $1 \%$ of patients requiring transfusion after THA. The results are reported as a consecutive series of cases from a single surgeon, therefore eliminating inter-surgeon variability. The results from this small study are promising, and suggests that topical application of TXA may be a way to significantly reduce the risk of exposure in total joint replacement patients to allogeneic blood. Further studies, including randomized controlled trials, are warranted to validate these initial findings.

\section{Supplementary Material}

Refer to Web version on PubMed Central for supplementary material.

\section{Acknowledgments}

Financial support: This research was supported by a grant from the National Institutes of Health (T32GM075770)

\section{References}

1. Bierbaum BE, Callaghan JJ, Galante JO, Rubash HE, Tooms RE, Welch RB. An analysis of blood management in patients having a total hip or knee arthroplasty. J Bone Joint Surg Am. Jan; 1999 81(1):2-10. [PubMed: 9973048]

2. Eubanks JD. Antifibrinolytics in major orthopaedic surgery. J Am Acad Orthop Surg. Mar; 2010 18(3):132-138. [PubMed: 20190103]

3. Henry DA, Carless PA, Moxey AJ, et al. Anti-fibrinolytic use for minimising perioperative allogeneic blood transfusion. Cochrane Database Syst Rev. 2011; 3:CD001886. [PubMed: 21412876]

4. Yang ZG, Chen WP, Wu LD. Effectiveness and safety of tranexamic acid in reducing blood loss in total knee arthroplasty: a meta-analysis. J Bone Joint Surg Am. Jul 3; 2012 94(13):1153-1159. [PubMed: 22623147]

5. Mannucci PM. Hemostatic drugs. N Engl J Med. Jul 23; 1998 339(4):245-253. [PubMed: 9673304]

6. Raveendran R, Wong J. Tranexamic acid reduces blood transfusion in surgical patients while its effects on thromboembolic events and mortality are uncertain. Evid Based Med. Aug 4.2012

7. Krohn CD, Sorensen R, Lange JE, Riise R, Bjornsen S, Brosstad F. Tranexamic acid given into the wound reduces postoperative blood loss by half in major orthopaedic surgery. Eur J Surg Suppl. Jul. 2003 (588):57-61. [PubMed: 15200045] 
8. Wong J, Abrishami A, El Beheiry H, et al. Topical application of tranexamic acid reduces postoperative blood loss in total knee arthroplasty: a randomized, controlled trial. J Bone Joint Surg Am. Nov 3; 2010 92(15):2503-2513. [PubMed: 21048170]

9. Good L, Peterson E, Lisander B. Tranexamic acid decreases external blood loss but not hidden blood loss in total knee replacement. Br J Anaesth. May; 2003 90(5):596-599. [PubMed: 12697586]

10. Nadler SB, Hidalgo JH, Bloch T. Prediction of blood volume in normal human adults. Surgery. Feb; 1962 51(2):224-232. [PubMed: 21936146]

11. Ker K, Edwards P, Perel P, Shakur H, Roberts I. Effect of tranexamic acid on surgical bleeding: systematic review and cumulative meta-analysis. BMJ. 2012; 344:e3054. [PubMed: 22611164]

12. Alvarez JC, Santiveri FX, Ramos I, Vela E, Puig L, Escolano F. Tranexamic acid reduces blood transfusion in total knee arthroplasty even when a blood conservation program is applied. Transfusion. Mar; 2008 48(3):519-525. [PubMed: 18067499]

13. Camarasa MA, Olle G, Serra-Prat M, et al. Efficacy of aminocaproic, tranexamic acids in the control of bleeding during total knee replacement: a randomized clinical trial. Br J Anaesth. May; 2006 96(5):576-582. [PubMed: 16531440]

14. Chareancholvanich K, Siriwattanasakul P, Narkbunnam R, Pornrattanamaneewong C. Temporary clamping of drain combined with tranexamic acid reduce blood loss after total knee arthroplasty: a prospective randomized controlled trial. BMC Musculoskelet Disord. 2012; 13:124. [PubMed: 22817651]

15. Garneti N, Field J. Bone bleeding during total hip arthroplasty after administration of tranexamic acid. J Arthroplasty. Jun; 2004 19(4):488-492. [PubMed: 15188109]

16. Imai N, Dohmae Y, Suda K, Miyasaka D, Ito T, Endo N. Tranexamic Acid for Reduction of Blood Loss During Total Hip Arthroplasty. J Arthroplasty. Jun 13.2012

17. Lee SH, Cho KY, Khurana S, Kim KI. Less blood loss under concomitant administration of tranexamic acid and indirect factor Xa inhibitor following total knee arthroplasty: a prospective randomized controlled trial. Knee Surg Sports Traumatol Arthrosc. Oct 2.2012

18. McConnell JS, Shewale S, Munro NA, Shah K, Deakin AH, Kinninmonth AW. Reduction of blood loss in primary hip arthroplasty with tranexamic acid or fibrin spray. Acta Orthop. Dec; 2011 82(6):660-663. [PubMed: 21999623]

19. Molloy DO, Archbold HA, Ogonda L, McConway J, Wilson RK, Beverland DE. Comparison of topical fibrin spray and tranexamic acid on blood loss after total knee replacement: a prospective, randomised controlled trial. J Bone Joint Surg Br. Mar; 2007 89(3):306-309. [PubMed: 17356139]

20. Zufferey P, Merquiol F, Laporte S, et al. Do antifibrinolytics reduce allogeneic blood transfusion in orthopedic surgery? Anesthesiology. Nov; 2006 105(5):1034-1046. [PubMed: 17065899]

21. Akizuki S, Yasukawa Y, Takizawa T. A new method of hemostasis for cementless total knee arthroplasty. Bull Hosp Jt Dis. 1997; 56(4):222-224. [PubMed: 9438084]

22. Ishida K, Tsumura N, Kitagawa A, et al. Intra-articular injection of tranexamic acid reduces not only blood loss but also knee joint swelling after total knee arthroplasty. Int Orthop. Nov; 2011 35(11):1639-1645. [PubMed: 21253725]

23. Onodera T, Majima T, Sawaguchi N, Kasahara Y, Ishigaki T, Minami A. Risk of deep venous thrombosis in drain clamping with tranexamic acid and carbazochrome sodium sulfonate hydrate in total knee arthroplasty. J Arthroplasty. Jan; 2011 27(1):105-108. [PubMed: 21435821]

24. Mutsuzaki H, Ikeda K. Intra-articular injection of tranexamic acid via a drain plus drain-clamping to reduce blood loss in cementless total knee arthroplasty. J Orthop Surg Res. Sep 29.2012 7(1): 32. [PubMed: 23020868]

25. Seo JG, Moon YW, Park SH, Kim SM, Ko KR. The comparative efficacies of intra-articular and IV tranexamic acid for reducing blood loss during total knee arthroplasty. Knee Surg Sports Traumatol Arthrosc. Jun 24.2012 


\section{Table 1}

Baseline demographic and clinical characteristics. Data reported as mean \pm SD. P values are for unpaired twotailed Student's T-test, except for gender, which is for Fisher's Exact test.

\begin{tabular}{cccc}
\hline \multicolumn{4}{c}{ Primary Hips } \\
\hline No TXA (n=40) & With TXA (n=91) & P value \\
\hline Gender (M/F) & $17 / 23$ & $45 / 46$ & 0.57 \\
Age & $59 \pm 13$ & $60 \pm 14$ & 0.86 \\
BMI & $33 \pm 10$ & $32 \pm 8$ & 0.51 \\
ASA & $2.7 \pm 0.6$ & $2.6 \pm 0.6$ & 0.33 \\
Preop Hgb & $14.0 \pm 1.3$ & $13.9 \pm 1.3$ & 0.58 \\
\hline \multicolumn{4}{c}{ Primary Knees } \\
\hline No TXA (n=29) & With TXA (n=130) & P value \\
\hline Gender (M/F) & $10 / 19$ & $51 / 79$ & 0.83 \\
Age & $61 \pm 10$ & $61 \pm 10$ & 0.75 \\
BMI & $34 \pm 8$ & $36 \pm 9$ & 0.37 \\
ASA & $2.8 \pm 0.5$ & $2.7 \pm 0.5$ & 0.33 \\
Preop Hgb & $13.8 \pm 1.5$ & $13.7 \pm 1.2$ & 0.57 \\
\hline
\end{tabular}




\section{Table 2}

Primary outcomes for TKA and THA patients. Data reported as mean \pm SD. P values are for unpaired twotailed Student's T-test, except for transfused \% which are for Fischer's Exact test.

\begin{tabular}{cccc}
\hline \multicolumn{4}{c}{ Primary Hips } \\
\hline & No TXA (n=40) & With TXA (n=91) & P value \\
\hline Length of stay (days) & $3.3 \pm 2.0$ & $2.7 \pm 0.7$ & 0.02 \\
Discharge Hgb (gm/dL) & $9.5 \pm 1.4$ & $10.5 \pm 1.4$ & $<0.01$ \\
Nadir in-hospital Hgb(gm/dL) & $9.4 \pm 1.3$ & $10.2 \pm 1.3$ & $<0.01$ \\
Hgb drop (gm/dL) & $4.7 \pm 1.2$ & $3.7 \pm 1.2$ & $<0.01$ \\
Blood loss (mL) & $1729 \pm 552$ & $1384 \pm 456$ & $<0.01$ \\
Transfused (\%) & $15 \%$ & $1 \%$ & $<0.01$ \\
\hline & Primary Knees & & \\
\hline Length of stay (days) & $3.1 \pm 0.9$ & $2.7 \pm 0.7$ & 0.01 \\
Discharge Hgb (gm/dL) & $10.5 \pm 1.8$ & $11.1 \pm 1.4$ & 0.05 \\
Nadir in-hospital Hgb(gm/dL) & $10.2 \pm 1.8$ & $10.7 \pm 1.3$ & 0.08 \\
Hgb drop (gm/dL) & $3.6 \pm 1.0$ & $3.0 \pm 0.9$ & $<0.01$ \\
Blood loss (mL) & $1397 \pm 473$ & $1166 \pm 390$ & 0.01 \\
Transfused (\%) & $10 \%$ & $0 \%$ & $<0.01$ \\
\hline
\end{tabular}

